\title{
Energy scale of the Big-Bounce
}

\author{
Włodzimierz Piechocki \\ Theoretical Physics Department, Institute for Nuclear Studies, \\ Hoża 69, 00-681 Warszawa, Poland
}

\begin{abstract}
We examine the nature of the cosmological big-bounce (BB) transition within the loop geometry underlying Loop Quantum Cosmology (LQC) at classical and quantum levels. Our canonical quantization method is an alternative to the standard LQC. Our method opens the door for analyzes of spectra of physical observables like energy density and volume operator. We find that one cannot determine the energy scale specific to BB by making use of the loop geometry without an extra input from observational cosmology.
\end{abstract}

Keywords: non-standard LQC, spectra of observables, big-bounce transition

PACS: $98.80 . \mathrm{Qc}, 04.60 . \mathrm{Pp}$

\section{INTRODUCTION}

Observational cosmology strongly suggests that the universe emerged from a state with extremely high energy densities of physical fields. Mathematical cosmology gives numerous examples of solutions with an initial Big-Bang singularity. This is why it is commonly believed that the cosmological singularity is a real feature of the Universe.

It seems that one can treat the problem of the singularity by making use of loop geometry: classical Big-Bang turns into quantum Big-Bounce transition. Thus, one may try to answer some interesting questions: What is the energy scale of the unification of general relativity (GR) with quantum physics? What is the structure of spacetime at semi-classical level (simply connected, foamy or discrete)? What is the origin of matter, non-gravitational fields and spacetime? What was before the Big-Bounce?

We try to address the first two questions in the context of the flat FRW universe with massless scalar field.

There are two methods of quantization of the cosmology models based on loop geometry: (i) standard loop quantum cosmology (LQC), based on the rule: 'first quantize, then impose constraints [1, 2], and (ii) non-standard LQC using the rule: 'first solve constraints, then quantize [3, 4]. In the former case, one believes that the classical Big-Bang is replaced by quantum Big-Bounce due to strong quantum effects at the Planck scale $[5,6,7]$. In the latter case, one shows that it is the modification of GR by loop geometry which is responsible for the resolution of the singularity and the energy density of matter at the Big-Bounce is unknown [8]. 


\section{CLASSICAL LEVEL}

The gravitational part of the GR Hamiltonian reads [3]

$$
H_{g}:=\int_{\Sigma} d^{3} x\left(N^{i} C_{i}+N^{a} C_{a}+N C\right)
$$

where $\Sigma$, space-like part of spacetime $R \times \Sigma ;\left(N^{i}, N^{a}, N\right)$, Lagrange multipliers; $\left(C_{i}, C_{a}, C\right)$ are Gauss, diffeomorphism and scalar constraints; $(a, b=1,2,3)$, spatial indices; $(i, j, k=1,2,3)$ internal $S U(2)$ indices. Constrains must satisfy the specific algebra. For flat FRW universe with massless scalar field (in some special gauge) the Hamiltonian (1) turns into [3]

$$
H_{g}=-\gamma^{-2} \int_{\mathscr{V}} d^{3} x N e^{-1} \varepsilon_{i j k} E^{a j} E^{b k} F_{a b}^{i},
$$

where $\gamma$, Barbero-Immirzi parameter; $\mathscr{V} \subset \Sigma$, elementary cell; $N$, lapse function; $\varepsilon_{i j k}$, alternating tensor; $E_{i}^{a}$, density weighted triad; $F_{a b}^{k}=\partial_{a} A_{b}^{k}-\partial_{b} A_{a}^{k}+\varepsilon_{i j}^{k} A_{a}^{i} A_{b}^{j}$, curvature of $S U(2)$ connection $A_{a}^{i} ; e:=\sqrt{|\operatorname{det} E|}$.

Modification of GR by loop geometry means replacement [1] of $F_{a b}^{k}$ by its approximation

$$
F_{a b}^{k}(\lambda)=\lim _{\mu \rightarrow \lambda}\left\{-2 \operatorname{Tr}\left(\frac{h_{i j}^{(\mu)}-1}{\mu^{2} V_{o}^{2 / 3}}\right) \tau^{k o} \omega_{a}^{i o} \omega_{a}^{j}\right\}
$$

and we have

$$
F_{a b}^{k}=\lim _{\lambda \rightarrow 0} F_{a b}^{k}(\lambda)
$$

where $V_{0}$ is the so-called fiducial volume. Holonomy of the connection around the square loop with sides length $\mu V_{0}^{1 / 3}$ is found to be

$$
h_{i j}^{(\mu)}=h_{i}^{(\mu)} h_{j}^{(\mu)}\left(h_{i}^{(\mu)}\right)^{-1}\left(h_{j}^{(\mu)}\right)^{-1}
$$

The holonomy along straight edge ${ }^{o} e_{k}^{a} \partial_{a}$ of length $\mu V_{0}^{1 / 3}$ (in fundamental, $\mathrm{j}=1 / 2$, representation of $\mathrm{SU}(2)$ group) reads

$$
h_{k}^{(\mu)}(c)=\exp \left(\tau_{k} \mu c\right)=\cos (\mu c / 2) 1+2 \sin (\mu c / 2) \tau_{k},
$$

where $\tau_{k}=-i \sigma_{k} / 2$ ( $\sigma_{k}$ are the Pauli spin matrices).

Making use of the so-called Thiemann identity leads finally to

$$
H_{g}=\lim _{\lambda \rightarrow 0} H_{g}^{(\lambda)}
$$

where

$$
H_{g}^{(\lambda)}=-\frac{s g n(p)}{2 \pi G \gamma^{3} \lambda^{3}} \sum_{i j k} N \varepsilon^{i j k} \operatorname{Tr}\left(h_{i}^{(\lambda)} h_{j}^{(\lambda)}\left(h_{i}^{(\lambda)}\right)^{-1}\left(h_{j}^{(\lambda)}\right)^{-1} h_{k}^{(\lambda)}\left\{\left(h_{k}^{(\lambda)}\right)^{-1}, V\right\}\right),
$$


and where $V=|p|^{\frac{3}{2}}=a^{3} V_{0}$ is the volume of the elementary cell $\mathscr{V}$. Variables $c$ and $p$ determine connections $A_{a}^{k}$ and triads $E_{k}^{a}: A_{a}^{k}={ }^{o} \omega_{a}^{k} c V_{0}^{-1 / 3}$ and $E_{k}^{a}={ }^{o} e_{k}^{a} \sqrt{q_{o}} p V_{0}^{-2 / 3}$, where $c=\gamma \dot{a} V_{0}^{1 / 3}$ and $|p|=a^{2} V_{0}^{2 / 3},\{c, p\}=8 \pi G \gamma / 3$.

Total Hamiltonian for FRW universe with a massless scalar field $\phi$ reads [3]

$$
H=H_{g}+H_{\phi},
$$

where $H_{g}$ is defined by (7) and $H_{\phi}=p_{\phi}^{2}|p|^{-\frac{3}{2}} / 2$, and where $\phi$ and $p_{\phi}$ are elementary variables satisfying $\left\{\phi, p_{\phi}\right\}=1$. The relation $H \approx 0$ defines the physical phase space.

Making use of (6), we calculate (8) and get the modified total Hamiltonian corresponding to (9)

$$
H^{(\lambda)} / N=-\frac{3}{8 \pi G \gamma^{2}} \frac{\sin ^{2}(\lambda \beta)}{\lambda^{2}} v+\frac{p_{\phi}^{2}}{2 v}
$$

where

$$
\beta:=\frac{c}{|p|^{1 / 2}}, \quad v:=|p|^{3 / 2}
$$

are the canonical variables (of so-called improved scheme). It should be emphasized that (10) presents a purely classical modified Hamiltonian (with no quantum corrections)!

The Poisson bracket for the canonical variables $\left(\beta, v, \phi, p_{\phi}\right)$ is defined to be

$$
\{\cdot, \cdot\}:=4 \pi G \gamma\left[\frac{\partial \cdot}{\partial \beta} \frac{\partial \cdot}{\partial v}-\frac{\partial \cdot}{\partial v} \frac{\partial \cdot}{\partial \beta}\right]+\frac{\partial \cdot}{\partial \phi} \frac{\partial \cdot}{\partial p_{\phi}}-\frac{\partial \cdot}{\partial p_{\phi}} \frac{\partial \cdot}{\partial \phi} .
$$

The dynamics of a canonical variable $\xi$ is

$$
\dot{\xi}:=\left\{\xi, H^{(\lambda)}\right\}, \quad \xi \in\left\{\beta, \nu, \phi, p_{\phi}\right\},
$$

where $\dot{\xi}:=d \xi / d \tau$, and where $\tau$ is an evolution parameter. Dynamics in physical phase space, $\mathscr{F}_{\text {phys }}^{(\lambda)}$, is defined by solutions to $(13)$ satisfying the condition $H^{(\lambda)} \approx 0$. Solutions of (13) ignoring the constraint $H^{(\lambda)} \approx 0$ are in kinematical phase space, $\mathscr{F}_{\text {kin }}^{(\lambda)}$.

If the Hamiltonian is a constraint which may be rewritten as a product of a simpler constraint and a function on $\mathscr{F}_{\text {kin }}^{(\lambda)}$ with no zeros, the original dynamics may be reduced to the dynamics with the simpler constraint [3]. Equation (10) can be rewritten as

$$
H^{(\lambda)}=N H_{0}^{(\lambda)} \tilde{H}^{(\lambda)} \approx 0
$$

where

$$
H_{0}^{(\lambda)}:=\frac{3}{8 \pi G \gamma^{2} v}\left(\kappa \gamma\left|p_{\phi}\right|+v \frac{|\sin (\lambda \beta)|}{\lambda}\right), \quad \tilde{H}^{(\lambda)}:=\kappa \gamma\left|p_{\phi}\right|-v \frac{|\sin (\lambda \beta)|}{\lambda},
$$

where $\kappa^{2} \equiv 4 \pi G / 3$.

$H_{0}^{(\lambda)}=0$ iff $p_{\phi}=0=\sin (\lambda \beta)$. In such case $\tilde{H}^{(\lambda)}=0$, thus $H^{(\lambda)}$ equals identically zero so there is no dynamics. We exclude such pathological cases from further considerations and assume that $H_{0}^{(\lambda)} \neq 0$. 
For functions $f$ and $g$ on $\mathscr{F}_{\text {phys }}^{(\lambda)}$ we have

$$
\begin{gathered}
\dot{f}=\left\{f, N H_{0}^{(\lambda)} \tilde{H}^{(\lambda)}\right\}=\left\{f, N H_{0}^{(\lambda)}\right\} \tilde{H}^{(\lambda)}+N H_{0}^{(\lambda)}\left\{f, \tilde{H}^{(\lambda)}\right\}=N H_{0}^{(\lambda)}\left\{f, \tilde{H}^{(\lambda)}\right\}, \\
\dot{g}=\left\{g, N H_{0}^{(\lambda)} \tilde{H}^{(\lambda)}\right\}=N H_{0}^{(\lambda)}\left\{g, \tilde{H}^{(\lambda)}\right\}, \quad \text { for } \quad \tilde{H}^{(\lambda)} \approx 0 .
\end{gathered}
$$

The relation

$$
\frac{\dot{f}}{\dot{g}}=\frac{d f}{d g}=\frac{N H_{0}^{(\lambda)}\left\{f, \tilde{H}^{(\lambda)}\right\}}{N H_{0}^{(\lambda)}\left\{g, \tilde{H}^{(\lambda)}\right\}}=\frac{\left\{f, \tilde{H}^{(\lambda)}\right\}}{\left\{g, \tilde{H}^{(\lambda)}\right\}}, \quad \text { as } \quad H_{0}^{(\lambda)} \neq 0,
$$

may be rewritten as [3]

$$
\frac{d f}{\left\{f, \tilde{H}^{(\lambda)}\right\}}=\frac{d g}{\left\{g, \tilde{H}^{(\lambda)}\right\}}
$$

Thus, in the relative dynamics one canonical variable may be used as an 'evolution parameter', and the dynamics is gauge independent (no dependance on $N$ ).

Since the relative dynamics is gauge independent, we choose $N=1 / H_{0}^{(\lambda)}$ to simplify calculations. Equations of motion read [3]

$$
\begin{gathered}
\dot{\phi}=\kappa \gamma \operatorname{sgn}\left(p_{\phi}\right), \quad \dot{p_{\phi}}=0, \\
\dot{\beta}=-4 \pi G \gamma \frac{|\sin (\lambda \beta)|}{\lambda}, \quad \dot{v}=4 \pi G \gamma \nu \cos (\lambda \beta) \operatorname{sgn}(\sin (\lambda \beta)), \\
\tilde{H}^{(\lambda)} \approx 0 .
\end{gathered}
$$

Solution of relative dynamics is found to be [3]

$$
2 v=\Delta \cosh \left(3 \kappa s\left(\phi-\phi_{0}\right)-\ln \Delta\right),
$$

where $s:=\operatorname{sgn}\left(p_{\phi}\right), \Delta:=\kappa \gamma \lambda\left|p_{\phi}\right|$. Solution for $\beta$ may be determined from (22) rewritten as [3]

$$
v|\sin (\lambda \beta)|=\kappa \gamma \lambda\left|p_{\phi}\right| .
$$

We can see that the variables $v$ and $\beta$ are functions of an evolution parameter $\phi$.

A function, $\mathscr{O}: \mathscr{F}_{\text {kin }}^{(\lambda)} \rightarrow R$, is a Dirac observable (we choose $N=1 / H_{0}^{(\lambda)}$ as our method is gauge invariant) if

$$
\left\{\mathscr{O}, H^{(\lambda)}\right\}=\left\{\mathscr{O}, N H_{0}^{(\lambda)} \tilde{H}^{(\lambda)}\right\}=\left\{\mathscr{O}, \tilde{H}^{(\lambda)}\right\}=0 .
$$

Thus $\mathscr{O}$ is solution to the equation [3]

$$
\frac{\sin (\lambda \beta)}{\lambda} \frac{\partial \mathscr{O}}{\partial \beta}-v \cos (\lambda \beta) \frac{\partial \mathscr{O}}{\partial v}-\frac{\kappa \gamma \operatorname{sgn}\left(p_{\phi}\right)}{4 \pi G} \frac{\partial \mathscr{O}}{\partial \phi}=0 .
$$

Solutions to (26) are found to be [3]

$$
\mathscr{O}_{1}:=p_{\phi}, \quad \mathscr{O}_{2}:=\phi-\frac{s}{3 \kappa} \operatorname{arth}(\cos (\lambda \beta)), \quad \mathscr{O}_{3}:=s v \frac{\sin (\lambda \beta)}{\lambda} .
$$


Observables satisfy the Lie algebra [3]

$$
\left\{\mathscr{O}_{2}, \mathscr{O}_{1}\right\}=1, \quad\left\{\mathscr{O}_{1}, \mathscr{O}_{3}\right\}=0, \quad\left\{\mathscr{O}_{2}, \mathscr{O}_{3}\right\}=\gamma \kappa .
$$

Due to the constraint $\tilde{H}^{(\lambda)}=0$, we have

$$
\mathscr{O}_{3}=\gamma \kappa \mathscr{O}_{1}
$$

Thus, in the physical phase space, $\mathscr{F}_{\text {phys }}^{(\lambda)}$, we have only two observables which satisfy the algebra

$$
\left\{\mathscr{O}_{2}, \mathscr{O}_{1}\right\}=1
$$

Our kinematical phase space, $\mathscr{F}_{\text {kin }}^{(\lambda)}$, is four dimensional. In relative dynamics one variable is used to parameterize three others. Since the constraint relates two variables, we have only two independent variables. This is the reason we have only two observables.

We consider functions which can be expressed in terms of observables and an evolution parameter $\phi$ so they are not observables. They do become observables for each fixed value of $\phi$, since in such case they are only functions of observables.

In what follows we consider the energy density of matter and the volume function. The energy density of matter field reads

$$
\rho(\lambda, \phi)=\frac{1}{2} \frac{p_{\phi}^{2}}{v^{2}} .
$$

In terms of observables we have [3]

$$
p_{\phi}=\mathscr{O}_{1}, \quad v=\kappa \gamma \lambda\left|\mathscr{O}_{1}\right| \cosh \left(3 \kappa\left(\phi-\mathscr{O}_{2}\right)\right)
$$

The density $\rho$ takes its maximum at the minimum of $v$

$$
\rho_{\max }=\frac{1}{2 \kappa^{2} \gamma^{2}} \frac{1}{\lambda}^{2} .
$$

Let us apply (33) to the Planck scale: $l_{P l}:=\sqrt{\hbar G / c^{3}} \sim 10^{-35} m ; \quad \rho_{P l}:=c^{5} / \hbar G^{2} \sim$ $10^{19} \mathrm{GeV}$. Equation (33) in terms of suitable units reads]

$$
\rho_{\max }=\frac{3 c^{2}}{8 \pi G \gamma^{2}} \frac{1}{\lambda^{2}} .
$$

Substituting $\lambda=l_{P l}$ into (34) gives $\rho_{\max } / \rho_{P l} \simeq 2,07$; for $\rho_{\max }=\rho_{P l}$ we get $\lambda \simeq 1,44 l_{P l}$. Surprisingly, the classical expression (34) fits the Planck scale.

The volume function reads: $V(\phi)=v$. It results from the constraint equation

$$
v|\sin (\lambda \beta)|=\kappa \gamma \lambda\left|p_{\phi}\right|
$$

\footnotetext{
${ }^{1}$ We use $\gamma \simeq 0.24$ determined in the black hole entropy calculations.
} 
that at the classical level the volume function is bounded from below [3]

$$
v_{\min }=\kappa \gamma \lambda\left|p_{\phi}\right|
$$

For $\lambda=0$ the minimum of $v$ vanishes! An interesting question is: Does the quantum volume operator have a non-zero minimum eigenvalue?

\section{QUANTUM LEVEL}

Our quantization method of constrained systems is simple enough to be fully controlled analytically. In the Schrödinger representation (since $\mathscr{O}_{1}, \mathscr{O}_{2} \in R$ ) we have

$$
\mathscr{O}_{2} \mapsto \widehat{\mathscr{O}}_{2}:=x, \mathscr{O}_{1} \mapsto \widehat{\mathscr{O}}_{1}:=-i \hbar \partial_{x},
$$

where $x \in R$, and where the carrier space is $L^{2}(R)$. Thus, the representation of (30) reads

$$
\left[\widehat{\mathscr{O}}_{2}, \widehat{\mathscr{O}}_{1}\right]=i \hbar
$$

The representation is essentially self-adjoint on $C_{0}^{\infty}(R) \subset L^{2}(R)$.

In the representation (37) the energy density operator reads [4]

$$
\rho \rightarrow \widehat{\rho}:=\frac{1}{2(\kappa \gamma \lambda)^{2} \cosh ^{2}[3 \kappa(\phi-x)]} .
$$

Solution to the eigenvalue problem

$$
\widehat{\rho} \psi=\rho\left(x_{0}\right) \psi
$$

for fixed value of $\phi$, is found to be [4]

$$
\psi(x)=\delta\left(x-x_{0}\right), \quad \rho\left(x_{0}\right)=\frac{1}{2(\kappa \gamma \lambda)^{2}} \frac{1}{\cosh ^{2}\left(3 \kappa\left(\phi-x_{0}\right)\right)} .
$$

Energy density has the same functional form as the classical one! The range of spectrum is: $\left(0, \frac{1}{2(\kappa \gamma \lambda)^{2}}\right)$. We can determine $\rho_{\max }$ if we know $\lambda$. But, $\lambda$ is a free parameter of our method! Thus, finding the critical density of matter corresponding to the Big-Bounce is an open problem [8].

Since we have $V=v$, where

$$
v=\kappa \gamma \lambda\left|\mathscr{O}_{1}\right| \cosh \left[3 \kappa\left(\phi-\mathscr{O}_{2}\right)\right]=\left|\kappa \gamma \lambda \mathscr{O}_{1} \cosh \left[3 \kappa\left(\phi-\mathscr{O}_{2}\right)\right]\right|=:|w|,
$$

we first examine the spectrum of $\widehat{w}$. The quantum operator corresponding to $w$ is defined as follows [4]

$$
\begin{gathered}
\widehat{w} \psi:=\kappa \gamma \lambda \frac{1}{2}\left(\widehat{\mathscr{O}}_{1} \cosh \left[3 \kappa\left(\phi-\widehat{\mathscr{O}}_{2}\right)\right]+\cosh \left[3 \kappa\left(\phi-\widehat{\mathscr{O}}_{2}\right)\right] \widehat{\mathscr{O}}_{1}\right) \\
=i \frac{\kappa \gamma \lambda \hbar}{2}\left(+2 \cosh [3 \kappa(\phi-x)] \frac{d}{d x}-3 \kappa \sinh [3 \kappa(\phi-x)]\right) \psi
\end{gathered}
$$


where $\psi \in D(\widehat{w})$. The solution to the eigenvalue problem of the operator $\widehat{w}$ reads [8]

$$
\begin{gathered}
\widehat{w} \psi_{b}=b \psi_{b}, \quad b \in R, \\
\psi_{b}(x):=\frac{\sqrt{\frac{3 \kappa}{\pi}} \exp \left(i \frac{2 b}{3 \kappa^{2} \gamma \lambda \hbar} \arctan e^{3 \kappa(\phi-x)}\right)}{\cosh ^{\frac{1}{2}} 3 \kappa(\phi-x)} .
\end{gathered}
$$

For the volume operator, corresponding to $V=v=|w|$, we have

$$
V \longrightarrow \hat{V} f_{b}=|\hat{w}| f_{b}:=|b| f_{b}
$$

Thus, the spectrum of the operator $\widehat{V}$ seems to be continuous, and $b=0$ is the smallest eigenvalue of $\hat{V}$. However, it turns out that the spectrum is discrete [4].

\section{MINIMUM LENGTH PROBLEM}

Determination of $\lambda$ by standard LQC means [6]:

- considering eigenvalue problem for the area operator, $\widehat{A r}=\widehat{|p|}$, in kinematical phase space of LQC: $\widehat{A r}\left|\mu>=\frac{4 \pi \gamma l_{p}^{2}}{3}\right| \mu|| \mu>=: \operatorname{ar}(\mu) \mid \mu>$, where $\operatorname{ar}(\mu)$ are continuous since $\mu \in R$;

- making reference to discrete eigenvalues, $\{0, \Delta, \ldots\}$, of kinematical $\widehat{A r}$ of LQG, where $\Delta:=2 \sqrt{3} \pi \gamma l_{p}^{2}$

- assuming: $\operatorname{ar}(\lambda) \equiv \Delta$, which leads to $\lambda=3 \sqrt{3} / 2$.

One assumes in the standard LQC that a surface cannot be squeezed to the zero value due to the existence in the universe of the minimal quantum of area. Physical justification for this assumption, offered by standard LQC, is doubtful because [9]:

- $\widehat{A r}$ was examined so far only in kinematical Hilbert space of LQG, i.e. spectrum of $\widehat{A r}$ ignores the algebra of constraints of LQG so it has no physical meaning;

- discrete spectrum of LQG was used to replace continuous spectrum of LQC (spectral discretization 'by hand');

- LQC is not a cosmological sector of LQG, but a quantization method inspired by LQG. Thus, LQC and LQG are models of different systems.

The assumption of the standard LQC that low-lying eigenvalue of the area operator of LQG determines the basic parameter of LQC is ad hoc [9, 10].

\section{CONCLUSIONS}

The modification of the classical Hamiltonian realized by making use of the loop geometry, parameterized by $\lambda$, turns Big-Bang into Big-Bounce. However, $\lambda$ is a free parameter to be determined. 
The determination of $\lambda$ is presently a great challenge. It seems that the FRW model is useless in this context; no eigenvalue of the volume operator is privileged. An extension of study to homogeneous (Bianchi I, ...) and isotropic (Lemaître, ...) models cannot probably change the situation. It seems that it is the observational cosmology which may bring some resolution to the problem. So far, the cosmic photons reveal no dispersion [11] up to the energy scale $5 \times 10^{17} \mathrm{GeV}$. One hopes that the primordial gravitational waves have made some imprints on the CMB spectrum so they may be used to determine the allowed range of values for the parameter $\lambda$.

\section{ACKNOWLEDGMENTS}

The author would like to thank the organizers for inspiring atmosphere at the Meeting and partial financial support.

\section{REFERENCES}

1. A. Ashtekar, M. Bojowald and J. Lewandowski, Adv. Theor. Math. Phys. 7, 233 (2003).

2. M. Bojowald, Living Rev. Rel. 8, 11 (2005).

3. P. Dzierzak, P. Malkiewicz and W. Piechocki, "Turning big bang into big bounce: Classical dynamics," arXiv:0907.3436 [gr-qc].

4. P. Malkiewicz and W. Piechocki, "Turning big bang into big bounce: Quantum dynamics," arXiv:0908.4029 [gr-qc].

5. A. Ashtekar, T. Pawlowski and P. Singh, Phys. Rev. Lett. 96, 141301 (2006).

6. A. Ashtekar, T. Pawlowski and P. Singh, Phys. Rev. D 73, 124038 (2006).

7. A. Ashtekar, T. Pawlowski and P. Singh, Phys. Rev. D 74, 084003 (2006).

8. P. Malkiewicz and W. Piechocki, Phys. Rev. D 80, 063506 (2009).

9. P. Dzierzak, J. Jezierski, P. Malkiewicz and W. Piechocki, "Conceptual issues concerning the Big Bounce," arXiv:0810.3172 [gr-qc].

10. M. Bojowald, Class. Quant. Grav. 26, 075020 (2009).

11. F. Aharonian et al., Phys. Rev. Lett. 101, 170402 (2008). 